\title{
Current status of the singlet-doublet dark matter model
}

\section{Tomohiro Abe*}

Institute for Advanced Research, Nagoya University, Furo-cho Chikusa-ku, Nagoya, Aichi, 464-8602 Japan

Kobayashi-Maskawa Institute for the Origin of Particles and the Universe, Nagoya University, Furo-cho Chikusa-ku, Nagoya, Aichi, 464-8602 Japan

E-mail: abetomo@kmi.nagoya-u.ac.jp

\section{Ryosuke Sato}

Deutsches Elektronen-Synchrotron (DESY), Notkestraße 85, D-22607 Hamburg, Germany

E-mail: ryosuke.sato@desy.de

Recent direct detectioon searches for dark matter give stringent bounds on the dark matter mass and couplings. One way to relax the constraint is to rely on pseudoscalar interactions with fermionic dark matter. We focus on the singlet-doublet dark matter model, which predicts the pseudoscalar interactions by $\mathrm{CP}$ violation in the dark sector. Due to the $\mathrm{CP}$ violation, electric dipole moments (EDMs) are predicted. We show that the model can be tested by the combination of the direct detection experiments and the measurement of the electron EDM.

The 4th KMI International Symposium (KMI2019)

18-20, February 2019

Nagoya, Japan

${ }^{*}$ Speaker. 


\section{Introduction}

The recent dark matter direct detection experiments give a string gent bound on dark matter model that explains the dark matter energy density by the freezeout mechanism. If a dark matter candidate is fermionic and it interacts with mediators by pseudoscalar interaction, $\bar{\psi}_{\mathrm{DM}} i \gamma^{5} \psi_{\mathrm{DM}} S$, then it is easy to evade the strong constraints from DM direct detection while keeping the success of the freezeout mechanism. This is because the pseudoscalar interaction predicts $s$-wave DM annihilation processes and velocity suppression of the DM-nucleon scattering processes. CP violation in dark sector can make this pseudoscalar interaction. In that case, the SM Higgs is a mediator and we do not need additional scalar fields as mediators.

\section{Setup}

The singlet-doublet model $[1,2,3]$ contains the $\mathrm{CP}$ violation in the dark sector and naturally predicts the pseudo-scalar interactions. The model contains a $Z_{2}$-odd gauge singlet Majorana fermion $\omega$ and a $Z_{2}$-odd $\mathrm{SU}(2)_{L}$ doublet Dirac fermion whose hypercharge is $\frac{1}{2},\left(\eta, \xi^{\dagger}\right)^{T}$. The Lagrangian contains the following terms.

$$
\mathscr{L} \supset-\frac{M_{1}}{2} \omega \omega-M_{2} \xi \eta-y \omega H^{\dagger} \eta-y^{\prime} \xi H \omega+\text { (h.c.), }
$$

where $H$ is the SM Higgs fields. There are four complex parameters and three of their phases can be rotated away by the field redefinition. However, there remains one complex phases, $\phi=$ $\operatorname{Arg}\left(M_{1}^{*} M_{2}^{*} y y^{\prime}\right)$. This is the CP phase in the dark sector. For $0<\phi<\pi$, we have pseudo-scalar interaction between dark matter particles and the SM Higgs boson. The dark matter particle is a mixture of the three neutral component of the singlet and the doublet fields. In the following analysis, the singlet field is the dominant component of the DM.

There are three features in this setup. First, we have both scalar and pseudoscalar interaction terms, $\bar{\psi} \psi h$ and $\bar{\psi} i \gamma^{5} \psi h$, where $\psi$ is the dark matter particle. There is no symmetry to forbid the scalar interaction terms, but there is a region where the scalar interaction is suppressed accidentally. Second, if the pseudoscalar interaction is large, the model predict the annihilation of DM pairs into the SM particles in the current universe. It produces $\gamma$ ray, positron, and so on. The Fermi-LAT experiment measures $\gamma$ ray and gives a constraint on dark matter model for $m_{\mathrm{DM}}<100 \mathrm{GeV}$. Third, EDMs are induced by the $\mathrm{CP}$ violation. Since the pseudoscalar coupling is also related to the $\mathrm{CP}$ violation, large pseudoscalar coupling is disfavored by EDM though the pseudoscalar coupling is essential to avoid the constraints from the direct detection experiments while keeping the freezeout scenario. We use the latest constrain on the electron EDM measured by ACME experiment [4].

\section{Result}

Figure 1 shows the current status and prospect of the singlet-doublet model. We find that the CP-conserving region ( $\phi=0$ or $\pi$ ) is severely constrained, and thus the CP-violation is essential for this model. The latest upper bound on the electron EDM gives a bound on the model, and it can cover the most of the region of the parameter space in future. As can be seen from the lower panel, 
the region with $m_{\mathrm{DM}} \sim m_{h} / 2$, so-called the Higgs funnel region, is only the possibility if null result is obtained by the XENONnT, LZ, and electron EDM measurements.

Figure 2 shows the current status and prospect for the Higgs funnel region. Even the Darwin project, which is a ultimate experiment for the DM direct detection experiment, can not cover all the region of the parameter space if the model contains a large $\mathrm{CP}$ violation in the dark sector. In that region, the electron EDM is larger than $\mathscr{O}\left(10^{-32}\right)$ e $\mathrm{cm}$. Therefore, we have a chance to test this model by the eEDM measurements in case that experiments reach to $d_{e}=\mathscr{O}\left(10^{-32}\right) \mathrm{e} \mathrm{cm} \mathrm{in}$ future even if $\sigma_{\mathrm{SI}}$ is very small at the Higgs funnel region.

\section{Summary}

The singlet-doublet model can avoid the strong constraint from the Xenon1T experiment while keeping the right amount of DM energy density by using the freezeout mechanism. The model is testable thanks to the correlation between the DM-nucleon scattering cross section and the electron EDM. The Higgs funnel region can be covered if the electron EDM experiment reach to $\mathscr{O}\left(10^{-32}\right)$ e cm.

\section{References}

[1] R. Mahbubani and L. Senatore, Phys. Rev. D 73, 043510 (2006) doi:10.1103/PhysRevD.73.043510 [hep-ph/0510064].

[2] F. D’Eramo, Phys. Rev. D 76, 083522 (2007) doi:10.1103/PhysRevD.76.083522 [arXiv:0705.4493 [hep-ph]].

[3] R. Enberg, P. J. Fox, L. J. Hall, A. Y. Papaioannou and M. Papucci, JHEP 0711, 014 (2007) doi:10.1088/1126-6708/2007/11/014 [arXiv:0706.0918 [hep-ph]].

[4] V. Andreev et al. [ACME Collaboration], Nature 562, no. 7727, 355 (2018). doi:10.1038/s41586-018-0599-8

[5] E. Aprile et al. [XENON Collaboration], Phys. Rev. Lett. 121, no. 11, 111302 (2018) doi:10.1103/PhysRevLett.121.111302 [arXiv:1805.12562 [astro-ph.CO]].

[6] A. Albert et al. [Fermi-LAT and DES Collaborations], Astrophys. J. 834, no. 2, 110 (2017) doi:10.3847/1538-4357/834/2/110 [arXiv:1611.03184 [astro-ph.HE]].

[7] The ATLAS collaboration [ATLAS Collaboration], ATLAS-CONF-2018-054.

[8] A. M. Sirunyan et al. [CMS Collaboration], doi:10.1016/j.physletb.2019.04.025 arXiv:1809.05937 [hep-ex].

[9] D. S. Akerib et al. [LUX-ZEPLIN Collaboration], arXiv:1802.06039 [astro-ph.IM].

[10] D. M. Kara, I. J. Smallman, J. J. Hudson, B. E. Sauer, M. R. Tarbutt and E. A. Hinds, New J. Phys. 14, 103051 (2012) doi:10.1088/1367-2630/14/10/103051 [arXiv:1208.4507 [physics.atom-ph]].

[11] D. Kawall, J. Phys. Conf. Ser. 295, 012031 (2011). doi:10.1088/1742-6596/295/1/012031 


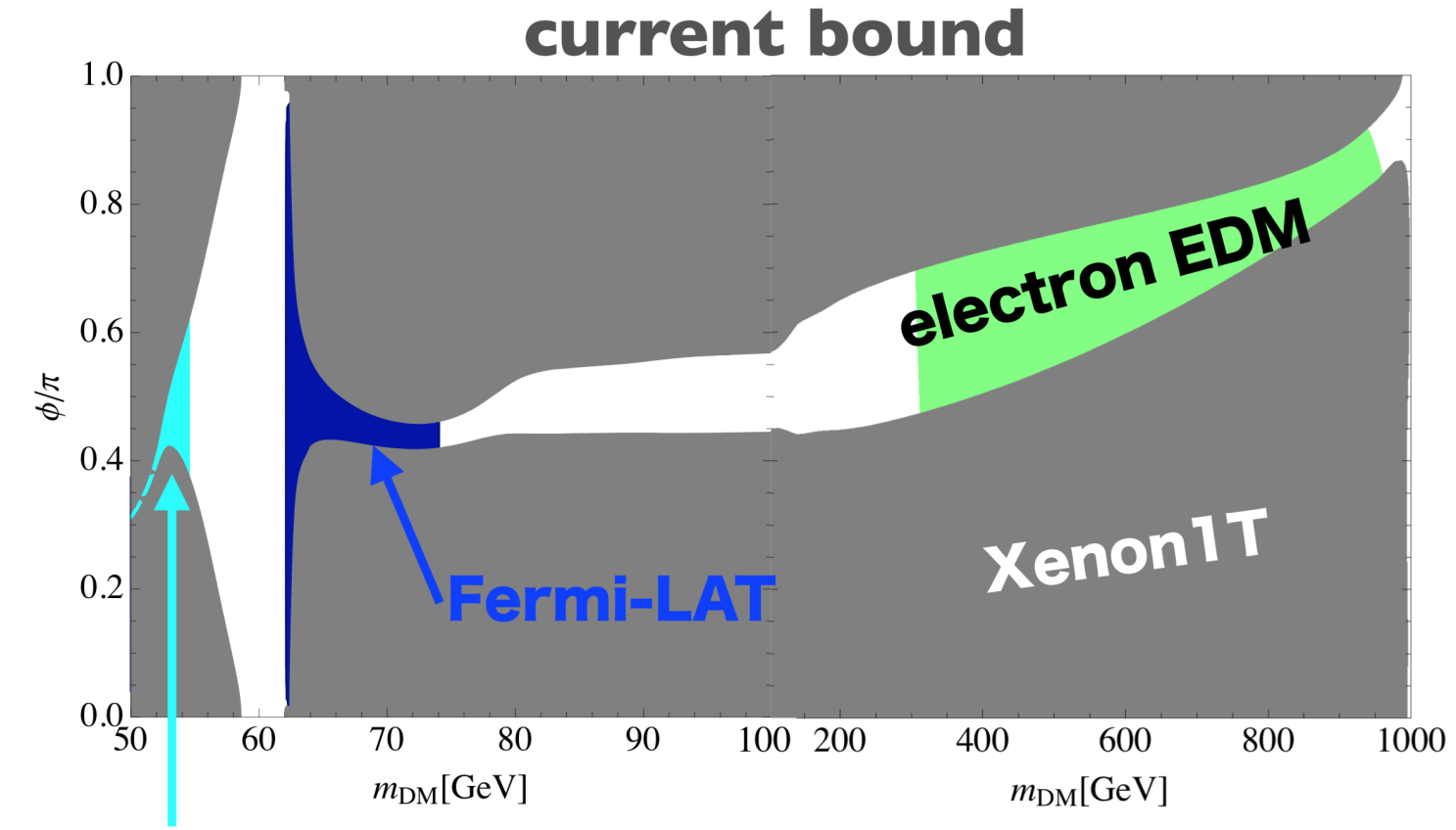

\section{Higgs invisible decay}

\section{prospects}

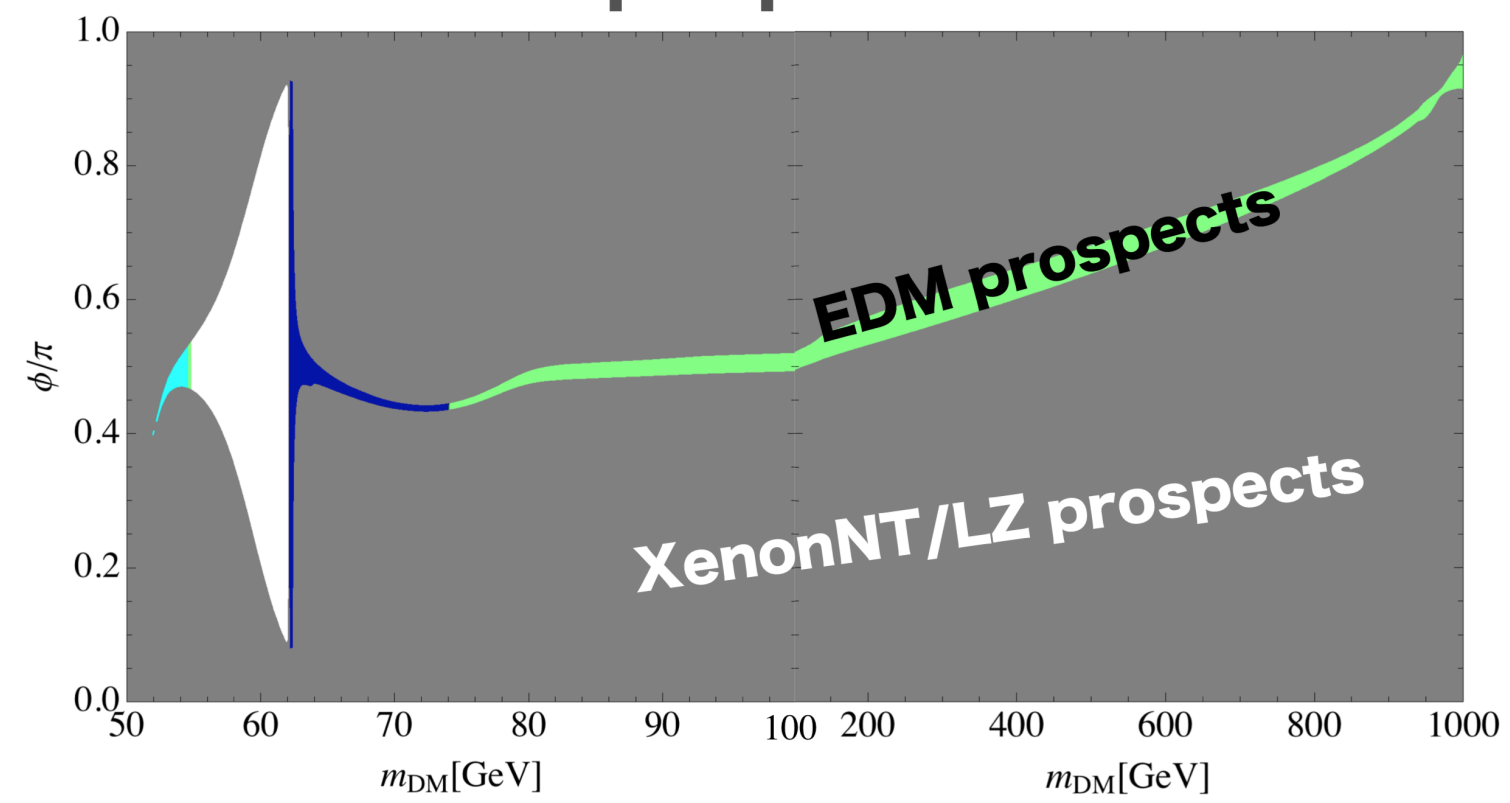

Figure 1: Current status (upper panels) and prospects (lower panels) of the singlet-doublet model for $M_{2}=$ $1 \mathrm{TeV}$ and $\left|y^{\prime}\right|=|y|$. The gray, green, and blue shaded regions are excluded by the XENON1T [5], ACME [4], and Fermi-LAT experiment [6], respectively. The cyan region is excluded by the measurement of the Higgs invisible decay at the LHC experiments [7, 8]. For the prospect, we assume null result and we use LZ [9] and $\left|d_{e}\right|=10^{-30} e \mathrm{~cm}[10,11]$. All the region in the figure explain the measured value of the dark matter energy density, $\Omega h^{2} \simeq 0.12$. 


\section{Higgs funnel region}
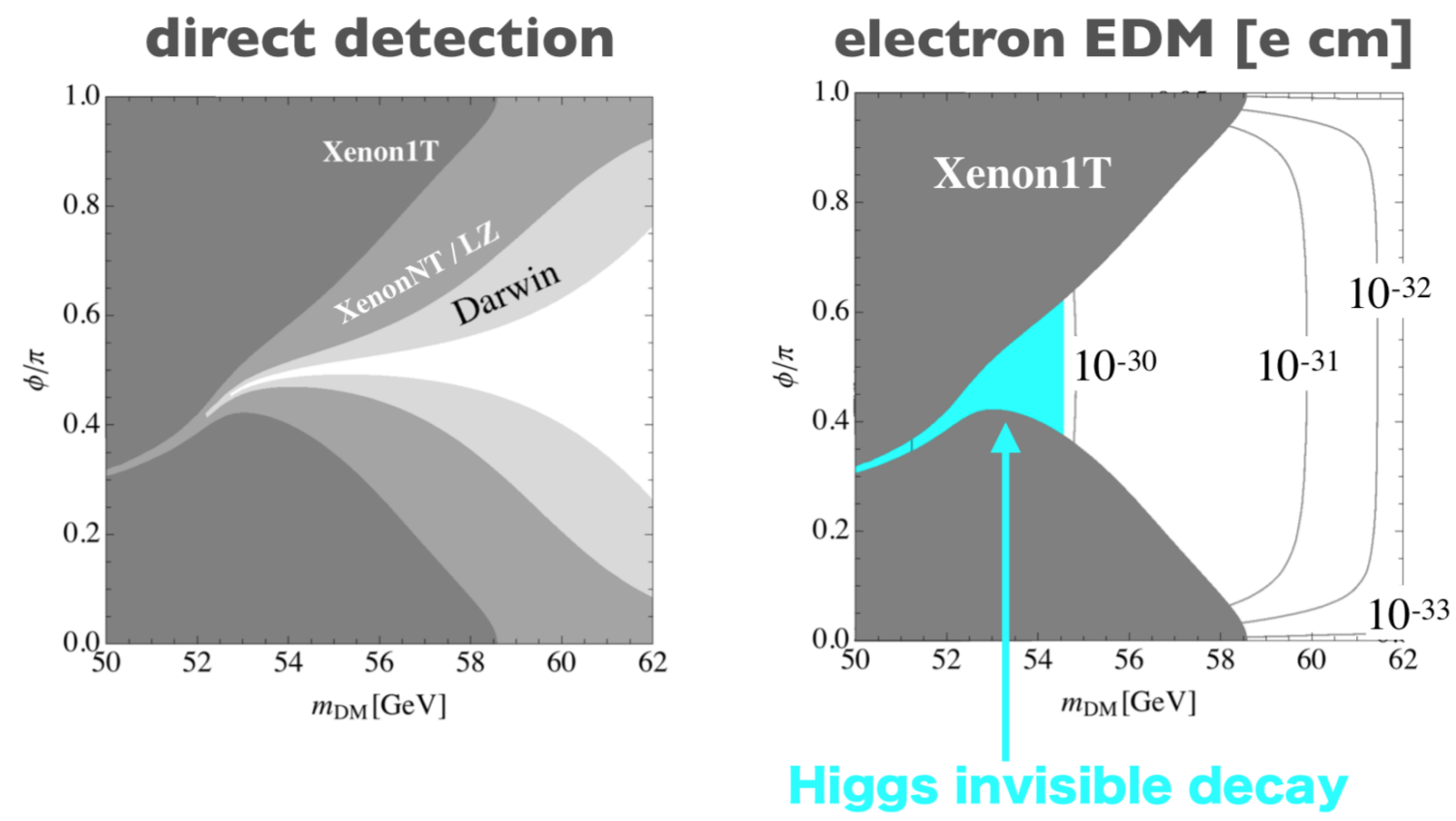

Figure 2: Left: The current bound and prospects of the direct detection experiments on the Higgs funnel region. Right: The eEDM contours for $M_{2}=1 \mathrm{TeV},|y|=\left|y^{\prime}\right|$, and $\phi=0.5 \pi$. The gray and cyan shaded regions are excluded by the XENON1T experiment [5], and the LHC experiment [7, 8], respectively. 